\title{
INDEPENDENCE OF THE PRIME IDEAL THEOREM FROM THE HAHN BANACH THEOREM
}

\author{
BY DAVID PINCUS
}

Communicated by A.H. Lachlan, February 1, 1972

1. Introduction. Principles in diverse areas of mathematics have been proven equivalent to the axiom of choice, AC. The same can be said of the prime ideal theorem for Boolean algebras, PI. AC is independent of PI by [2]. The Hahn Banach theorem, HB, is a consequence of PI [3], [7] and also has some surprising equivalent forms [4].

THEOREM 1. PI is independent of $H B$ in (the usual) $Z F$ set theory.

The Krein Milman theorem, $\mathrm{KM}$, is a consequence of $\mathrm{AC}$ and $\mathrm{PI}+\mathrm{KM} \rightarrow \mathrm{AC}^{1}$ This, together with the result of [1], shows $\mathrm{HB}+$ $\mathrm{VKM} \rightarrow \mathrm{AC}$ where VKM is a strengthened version of $\mathrm{KM}$. Let ZFA be the weakening of $\mathrm{ZF}$ to permit the existence of a set of atoms.

THEOREM 2. $A C$ is independent of $H B+K M$ in $Z F A$.

It is open whether ZF can replace ZFA in Theorem 2. We are grateful to W.A.J. Luxemburg who helped state these results in their present form.

2. The model with atoms. We use the original permutation model of Fraenkel. This model, and variants of it, is discussed by Mostowski in [5]. We do not give the construction of the model here. Instead we list some statements which are true in the model and base our subsequent arguments on these statements. The statements follow easily from observations of [5] and [6].

1. The axioms of ZFA hold.

2. There is a function $P$ on $\omega$ such that the $P_{i}$ are mutually disjoint pairs and $\mathrm{K}=\bigcup_{i \in \omega} P_{i}$ is the set of atoms.

3. There is a relation $\nabla(n, x)$, also written $x \in \nabla_{n}$, which satisfies:

(a) Every set is in some $\nabla_{n}, n \in \omega$.

(b) Each $\nabla_{n}$ contains all ordinals, the function $P$, and the members of $\bigcup_{i<n} P_{i}$

AMS 1970 subject classifications. Primary 02K05; Secondary 46A05.

Key words and phrases. Independence, Hahn Banach theorem, prime ideal theorem, Krein Milman theorem.

1 This result has been independently proved by Peter Renz, W.A.J. Luxemburg, and (jointly) J.L. Bell, and D.H. Fremlen. Peter Renz communicated the result to us. 
(c) If $\mathrm{y}$ is definable from parameters in $\nabla_{n}$, then $y \in \nabla_{n}$.

(d) If $m<n$ then $\nabla_{m} \subset \nabla_{n}$ and $\nabla_{m} \cap P_{n}=\varnothing$.

(e) If $y \subset \nabla_{n}$ and $y$ is a set then $y \in \nabla_{n}$.

4. There is a relation $T(Q, \alpha, x)$ such that if $Q$ is a choice function on $\left\{P_{i}\right\}_{i<n}$ then $T_{Q}=\{(\alpha, x): T(Q, \alpha, x)\}$ is a $1: 1$ function from the ordinals onto $\nabla_{n}$.

We now argue in the model from 1-4.

$P I$ is false.

Proof. PI implies the ordering principle which implies choice for pairs. On the other hand, as is shown in [5], choice for pairs is false. We can see this since a choice function, $g$, on $\left\{P_{i}\right\}_{i \in \omega}$ must be in some $\nabla_{n}$. $g\left(P_{n+1}\right)$ is a member of $P_{n+1}$ defined from parameters $(g, P$, and $n+1)$ in $\nabla_{n}$, contradicting $3(\mathrm{c})$ and $(\mathrm{d})$.

Lemma 1. Let $(X,+$,$) be a real vector space with (X,+, \cdot) \in \nabla_{n}$. Let $f: X \cap \nabla_{n} \rightarrow R$ be a linear functional. $f$ has a unique extention to a linear functional $g: X \rightarrow R$ such that $g \in \nabla_{n}$.

Proof. Notice that $X \cap \nabla_{m}$ is a subspace of $X$ for $m \geqq n$. If $x, y \in X \cap \nabla_{m}$ and $c, d \in R$ then $c \cdot x+d \cdot y$ is defined from parameters in $\nabla_{n}$. Since $n \leqq m \rightarrow \nabla_{n} \subset \nabla_{m}$ it suffices by induction to show that $f$ has a unique extention in $\nabla_{n}$ to $X \cap \nabla_{n+1}$.

Let $[x]_{n}$ denote the intersection of all sets in $\nabla_{n}$ which contain $x$. $[x]_{n} \in \nabla_{n}$ using 3(e). If $x \in \nabla_{n+1}$ then $[x]_{n}$ has at most two elements since, for some choice function $Q_{0}$ on $\left\{P_{i}\right\}_{i<n}$ and some $\alpha$,

$$
\delta=\left\{T_{Q}(\alpha): \operatorname{Dom} Q=\left\{P_{i}\right\}_{i<n+1} \wedge Q \supset Q_{0}\right\}
$$

is a term of the intersection. By 3(c) $x \in \nabla_{n} \leftrightarrow[x]_{n}=\{x\}$ so it is clear that for any $Q_{0}$ on $\left\{P_{i}\right\}_{i<n}$ there is some $\alpha$ such that $[x]_{n}$ has the form $\delta$.

Define ${ }^{*}: \nabla_{n+1} \rightarrow \nabla_{n+1}$ by $x^{*}=x$ for $x \in \nabla_{n}$ and $x^{*} \in[x]_{n}-\{x\}$ otherwise. If $x, y \in \nabla_{n+1}$ then $x \in y \rightarrow x^{*} \in y^{*}$ since otherwise there are $\alpha$ and $\beta$ such that some member of $P_{n}$ is defined as: "That $a \in P_{n}$ such that $T_{Q_{0} \cup\left(P_{n}, a\right)}(\alpha) \in T_{Q_{0} \cup\left(P_{n}, a\right)}(\beta)$." Manifestly $x^{* *}=x$ so ${ }^{*}$ is an $\varepsilon$ automorphism of $\nabla_{n+1}$ with fixed subclass $\nabla_{n} .{ }^{*}$ is therefore an automorphism of $X \cap \nabla_{n+1}$. Also $g(x)=g\left(x^{*}\right)$ for any real valued function $g \in \nabla_{n}$ on $X \cap \nabla_{n+1}$.

Uniqueness of $g$. If $x \in X \cap \nabla_{n+1}$ then $\left(x+x^{*}\right)^{*}=x^{*}+x^{* *}=x^{*}+x=$ $x+x^{*}$ and $x+x^{*} \in \nabla_{n}$. If $g \in \nabla_{n}$ is a linear extension of $f: g(x)=$ $\frac{1}{2}(g(x)+g(x))=\frac{1}{2}\left(g(x)+g\left(x^{*}\right)\right)=g\left(\frac{1}{2}\left(x+x^{*}\right)\right)=f\left(\frac{1}{2}\left(x+x^{*}\right)\right)$.

Existence of $g$. Define $g(x)=f\left(\frac{1}{2}\left(x+x^{*}\right)\right) . \quad f \in \nabla_{n}$ by $3(\mathrm{e})$ and $\frac{1}{2}\left(x+x^{*}\right)$ depends only on $[x]_{n}$ so $g \in \nabla_{n}$. 


$$
\begin{aligned}
g(c x+d y) & =f\left(\frac{1}{2}\left(c x+d y+(c x+d y)^{*}\right)\right) \\
& =f\left(\frac{1}{2}\left(c x+d y+c x^{*}+d y^{*}\right)\right) \\
& =f\left(c\left(\frac{1}{2}\left(x+x^{*}\right)\right)+d\left(\frac{1}{2}\left(y+y^{*}\right)\right)\right) \\
& =c f\left(\frac{1}{2}\left(x+x^{*}\right)\right)+d f\left(\frac{1}{2}\left(y+y^{*}\right)\right) \\
& =c g(x)+d g(y) .
\end{aligned}
$$

LEMMA 2. If, in Lemma $1,|f| \leqq p$ for a sublinear positively homogeneous functional $p$ on $X$ with $p \in \nabla_{n}$, then $|g| \leqq p$.

Proof. Again by induction from $n$ to $n+1$,

$$
\begin{aligned}
|g(x)|= & \left|f\left(\frac{1}{2}\left(x+x^{*}\right)\right)\right| \leqq p\left(\frac{1}{2}\left(x+x^{*}\right)\right)=\frac{1}{2} p\left(x+x^{*}\right) \\
& \leqq \frac{1}{2}\left(p(x)+p\left(x^{*}\right)\right)=\frac{1}{2}(p(x)+p(x))=p(x) .
\end{aligned}
$$

$p\left(x^{*}\right)=p(x)$, since $p \in \nabla_{n}$.

$H B$ is true.

Proof. Let $(X,+, \cdot)$ be a vector space, let $Y \subset X$ be a subspace, let $p$ be sublinear and positively homogeneous $X \rightarrow R$, and let $h: Y \rightarrow R$ be linear and satisfy $|h| \leqq p$. Choose an $n$ large enough so that $\nabla_{n}$ contains $(X,+), Y,$,$p , and h$. Let $f=h \uparrow Y \cap \nabla_{n}$. Since $X \cap \nabla_{n}$ can be well ordered there is an extension $f^{*}$ of $f$ to $X \cap \nabla_{n}$ which satisfies $\left|f^{*}\right| \leqq p$ (this is the usual HB). By Lemmas 1 and 2, there is an extension $g$ of $f^{*}$ to $X$ with $g \in \nabla_{n}$ and $|g| \leqq p . g\left\lceil Y\right.$ is in $\nabla_{n}$ and agrees with $h$ on $Y \cap \nabla_{n}$. Thus $g\lceil Y=h$ by uniqueness and HB is proved.

KM is true.

Proof. In view of $\mathrm{HB}$, and the usual proof of KM, we need only show that a convex, compact, nonempty set in a locally convex vector space has an extreme point. Note that if $(X,+, \cdot, \mathscr{T})$ is locally convex, $\varnothing \neq C \subset X$ convex and compact, and both are in $\nabla_{n}$ then $C \cap \nabla_{n} \neq \varnothing$. Inductively if $C \cap \nabla_{n+1} \neq \varnothing$ and $x \in C \cap \nabla_{n+1}$ then $\frac{1}{2}\left(x+x^{*}\right) \in C \cap \nabla_{n}$.

Define, as usual, a facet $\varnothing \neq F \subset C$ to be compact, convex, and satisfy

$$
\left(\frac{1}{2}(x+y) \in F \wedge x, y \in C\right) \rightarrow x, y \in F .
$$

The family of facets in $\nabla_{m}, m \geqq n$, satisfy the hypotheses of Zorn's lemma by $3(e)$, since a nested family of nonempty compact sets has the finite intersection property. $\nabla_{m}$ is well orderable so there is a facet of $C$ which is minimal among those in $\nabla_{m}$.

If $F$ is a $\nabla_{m}$-minimal facet of $C$ we have noted that $F \cap \nabla_{m} \neq \varnothing$. We now remark that $F \cap \nabla_{m}$ is a singleton. Two points in $F \cap \nabla_{m}$ can be separated by a linear functional which, by our proof of $\mathrm{HB}$, will be in 
$\nabla_{m}$. This functional takes its maximum on a proper subfacet of $F$ by the usual proof of KM. The subfacet will be in $\nabla_{m}$ by 3(c), a contradiction.

Using a well ordering of $\nabla_{n}$ we choose sets $\mathscr{F}_{m}=[F]_{n}$ for $m>n$ such that $F$ is a $\nabla_{m}$-minimal facet of $C$ and, for $m>n$, a subfacet of a member of $\mathscr{F}_{m-1}$. Every $G \in \mathscr{F}_{m}$ has the same property as $F$; otherwise one could find a smaller $\nabla_{m}$-set containing $F$. Similarly every $F \in \mathscr{F}_{m}$ has a subfacet in $\mathscr{F}_{m+1} . \mathscr{F}_{m}$ is finite, in fact, $\left|\mathscr{F}_{m}\right| \leqq 2^{m-n}$ as in Lemma 1.

The sequence $\left\{\bigcup \mathscr{F}_{m}\right\}_{m \geqq n}$ is a decreasing sequence of nonempty compact sets. Let $y$ be in its intersection. We claim that

$$
\bigcap\left\{F: y \in F \wedge(\exists m)\left(F \in \mathscr{F}_{m}\right)\right\}=\{y\}
$$

If $z$ is also in this intersection let $m$ be large enough so that $y, z \in \nabla_{m}$. $y$ and $z$ are both in some facet, $F$, of $\mathscr{F}_{m}$ so $y, z \in F \cap \nabla_{m}$ and, as was remarked, $y=z$. Thus $\{y\}$ is an intersection of facets so $\{y\}$ is a facet and $y$ is extremal.

3. Sketch of the ZF model. The model with atoms is embedded into a ZF model according to Theorem 4.2 of [6]. Using ideas of [2] and [6] we can show that the following statements hold in the model. 1 holds with ZFA replaced by ZF. 2 holds, but $K$ is no longer a set of atoms. 3(a),(b),(c), and (d) hold. The following modification of 4 holds.

$4^{\prime}$. $T_{Q}$ (as in 4) is a function on the class of Boolean algebras, $\mathscr{B}=(B, \sim, \wedge)$ with $\mathscr{B} \in \nabla_{n}$ and $B \subset \nabla_{n}$. For each such $\mathscr{B}, T_{Q}(\mathscr{B})$ is a prime ideal of $\mathscr{B}$ in $\nabla_{n}$.

PI is proven false as in $\$ 2$. The use of $3(\mathrm{e})$ must be circumvented in the proof of HB. Let $X,+, ;, p, Y$, and $f$ be as in the hypotheses of HB and assume that these are all in some $\nabla_{n}$. Inductively the problem boils down to extending $f$ canonically from $Y$ to $Y+\left(X \cap \nabla_{m}\right)$ for $m \geqq n$. The idea is to use $4^{\prime}$ to associate to each $Q$ (extending a fixed $Q_{0}$ for $\left.\left\{P_{i}\right\}_{i<n}\right)$ an extention $g_{Q}$. The dependence on $Q$ is removed by con$\left(1 / 2^{m-n}\right) \sum_{Q \supset Q_{0}} g_{Q}$.

$4^{\prime}$ can be used as in [3] to extend $f$ on $Y \cap \nabla_{m}$ to $g_{Q}$ on $X \cap \nabla_{m}$. The problem is to be sure that $g_{Q}$ continues to satisfy $\left|g_{Q}\right| \leqq p$ when extended to $Y+\left(X \cap \nabla_{m}\right)$. The usual proof of HB gives functions $\mu_{1}$ and $\mu_{2}$ on $X$ such that for an arbitrary linear functional $g \supset f,|g| \leqq p$ on $S p(x)+Y$ if and only if $\mu_{1}(x) \leqq g(x) \leqq \mu_{2}(x)$. A careful use of the methods of [3] will guarantee that $g_{Q}$ satisfies $\mu_{1} \leqq g \leqq \mu_{2}$ on $X \cap \nabla_{m}$. This proves HB.

The falsity of KM in our ZF model can easily be deduced from the falsity of KM in the model of [2], which, in turn, follows from PI + KM $\rightarrow$ AC. If Theorem 2 is to be extended to ZF a different model is necessary. 


\section{BIBLIOGRAPHY}

1. J. L. Bell and F. Jellett, On the relationship between the Boolean prime ideal theorem and two principles in functional analysis, Bull. Acad. Polon. Sci. Sér. Sci. Math. Astronom. Phys. 19 (1971), 191-194.

2. J. D. Halpern and A. Levy, The Boolean prime ideal does not imply the axiom of choice, Proc. Sympos. Pure Math., vol. 18, part 1, Amer. Math. Soc., Providence, R.I., 1970, pp. 83-134.

3. W. A. J. Luxemburg, Two applications of the method of construction by ultrapowers to analysis, Bull. Amer. Math. Soc. 68 (1962), 416-419. MR 25 \#3837.

4. - Reduced powers of the real number system and equivalents of the Hahn Banach extension theorem, Internat. Sympos. on Applications of Model Theory to Algebraic Analysis, and Probability, Holt, Rinehart and Winston, New York, 1969.

5. A. Mostowski, Axion of choice for finite sets, Fund. Math. 33 (1945), 137-168. MR 8 , 3.

6. D. Pincus, Support structures for the axiom of choice, J. Symbolic Logic 36 (1971), 28-38.

7. J. Kos and C. Ryll-Nardzewski, On the applications of Tychonoff's theorem in mathematical proofs, Fund. Math. 38 (1951), 233-237. MR 14, 70. 98105

Department of Mathematics, University of Washington, Seattle, Washington 